\title{
Dénombrement des spores de Clostridium tyrobutyricum par fiitration sur membrane et culture sur milieu gélosé
}

\author{
par \\ Brigitte ABGRALL et C. BOURGEOIS \\ avec la collaboration technique de Fabienne BOURVA
}

\section{Rés u mé}

Ce travail s'insère dans un ensemble de recherches dont le but est de substituer, à la technique actuelle de dénombrement des spores de $C$. tyrobutyricum dans le lait, une méthode répondant mieux aux exigences de sensibilité et de rapidité.

Un procédé de filtration original, publié par ailleurs, permet de concentrer sur une membrane de $47 \mathrm{~mm}$ de diamètre les spores contenues dans 100 à $400 \mathrm{ml}$ de lait, et donc d'obtenir une sensibilité suffisante.

L'amélioration de la rapidité de détection a été obtenue par utilisation d'un milieu de base peu sélectif, le RCM.

Ce milieu a été ensuite rendu partiellement sélectif par addition de cyclosérine qui inhibe les Bacillus et différentiel par addition de fuchsine acide ; l'incubation sur ce milieu en anaérobiose de la membrane filtrante permet de reconnaître et de dénombrer spécifiquement les colonies de $C$. tyrobutyricum.

La méthode a été testée avec succès sur des laits contaminés artificiellement. Toutefois son application effective dans de bonnes conditions nécessite divers travaux complémentaires.

Mots clés

Clostridium tyrobutyricum - Lait - Dénombrement.

A.D.R.I.A. (Association pour le Développement de la Recherche Appliquée aux Industries Agricoles et Alimentaires), 6, rue de l’Université, B.P. 313 - 29191 Quimper cedex. 


\section{S u m m a ry}

\section{ENUMERATION OF CLOSTRIDIUM TYROBUTYRICUM SPORES BY MEMBRANE FILTRATION AND CULTURE ON GELOSE MEDIA}

This work is a part of a comprehensive research program whose purpose is to replace the current technique for counting spores of C. tyrobutyricum in milk by another method, meeting better the industry requirements for sensivity and rapidity.

An original filtration process, published elsewhere, permits to collect, on a $47 \mathrm{~mm}$ diameter filter, spores contained in 100 to $400 \mathrm{ml}$ of milk and thus to obtain a sufficient sensivity.

The improvement of rapidity of detection has been obtained by utilization of a less selective base medium, the RCM.

This medium has then been rendered partially selective by addition of cyscloserine which inhibits Bacilli and differential by addition of acid fuchsin which allows to recognize and number specifically C. tyrobutyricum colonies, after incubation of the membrane filter on RCM medium anaerobically.

This method has been tested successfully with artificially contaminated milks. Nevertheless its actual application in good conditions requires further work.

Key words

Clostridium tyrobutyricum - Milk - Counting.

\section{INTRODUCTION}

Clostridium tyrobutyricum est l'agent principal du gonflement tardif des fromages de type Emmental ; quelques centaines de spores par litre de lait suffisent pour faire apparaître des défauts (Gouet et Bergère, 1973). Actuellement, le contrôle de la teneur des laits en spores de "butyriques » s'effectue par la méthode préconisée par l'Institut Technique du Gruyère (I.T.G.) sur le milieu de Bryant Burkey modifié par Bergère, par la technique du nombre le plus probable (Bergère et coll., 1972). Mais cette méthode est lente (5 à 7 jours), peu sensible et peu précise, dans les conditions courantes d'utilisation; elle n'est, de plus, pas spécifique de C. tyrobutyricum.

Pour pallier en partie ces inconvénients, nous avons proposé de concentrer les spores par filtration du lait (Bourgeois et coll., 1984) et de dénombrer les colonies obtenues sur le filtre, appliqué sur un milieu gélosé sélectif, après un temps d'incubation suffisant.

L'objet de ce travail était précisément la mise au point du milieu gélosé nécessaire. 


\section{MATERIEL ET METHODES}

\section{Micro-organismes tests}

Clostridium tyrobutyricum (6 souches ; C.N.R.Z. Jouy-en-Josas), Clostridium sporogenes (I.P. 60.53), Clostridium bifermentans (I.P. 60.14), Bacillus licheniformis (C.N.R.Z. Jouy-en-Josas), Bacillus cereus (C.N.R.Z. Jouy-en-Josas).

Les Clostridium sont conservés en bouillon RCM (Merck, 5410), les Bacillus sur gélose de Wang (Wang et coll., 1964).

Les deux espèces de Bacillus choisies sont les plus fréquentes dans le lait (Bergère, C.P.). Les espèces de Clostridium les plus fréquemment rencontrées dans le lait sont : C. perfringens, C. butyricum (Goudkov et Sharpe, 1965 ; Cerf et Bergère, 1968) et surtout $C$. sporogenes (Bergère, 1983). C. bifermentans est signalé dans les ensilages (Gibson, 1965) et dans les fromages altérés (Prévot, 1953 cité par Goudkov et Sharpe, 1965). C. perfringens et $C$. butyricum n'ont pas été testés, les souches dont nous disposions n'ayant pas sporulé.

\section{Obtention des suspensions de spores}

Les spores de Clostridium ont été obtenues par culture sur milieu TGE (Bergère et Hermier, 1965) par la méthode des cultures biphasiques. Après centrifugation et lavage, les spores ont été conservées en eau distillée stérile.

Les spores de Bacillus ont été obtenues sur une gélose de Wang ; après incubation de la culture pendant 1 semaine à $30^{\circ} \mathrm{C}$, les spores ont été récoltées et conservées en eau distillée stérile.

\section{Détermination de la sensibilité des germes tests à différents agents sélectifs}

Différents antibiotiques et colorants, choisis parmi les plus couramment utilisés dans les milieux de culture, ont été testés sur un milieu de base constitué par le RCM Agar Merck préparé et stérilisé selon les indications du fabricant. Les colorants ont été incorporés directement au milieu sec; les solutions d'antibiotiques préparées extemporanément et stérilisées par filtration ont été ajoutées au milieu de base fondu et thermostaté à $45^{\circ} \mathrm{C}$. Le milieu complet a été coulé en boîtes de Pétri (diamètre $55 \mathrm{~mm}$ ) et séché sous hotte à flux laminaire.

Les suspensions de spores ont été filtrées sous vide sur membranes en esters de cellulose (Millipore AAWP 04700). Les membranes ont été déposées sur le milieu et incubées $48 \mathrm{~h}$ à $37^{\circ} \mathrm{C}$ en jarre anaérobie (système Anaérocult A - Merck). 
Après incubation, le développement des germes tests en présence des agents sélectifs a été comparé, par dénombrement des colonies obtenues sur la membrane, à un témoin réalisé sur le milieu de base (RCM sans agent sélectif).

\section{Filtration sous pression du lait}

Elle s'effectue conformément au protocole suivant (Bourgeois et coll., 1984).
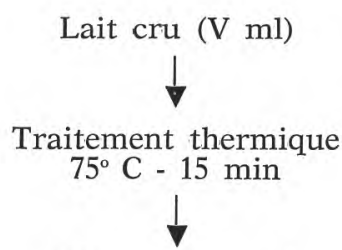

Refroidissement<smiles>C[13CH]</smiles>

Addition de :

Triton X-100 à $1 \%$ en eau distillée (V ml)

Trypsine à $1 \%$ en tampon Tris $\mathrm{HCl} 0,1 \mathrm{M}$ à pH 7,8 (V/4 ml)

Homogénéisation au Stomacher pendant $1 \mathrm{~min}$

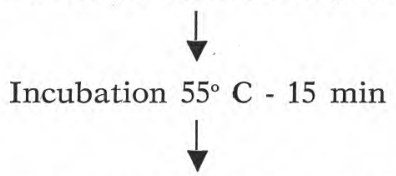

Filtration à 3 bars sur membrane 0,8 micromètre

Rinçage de la membrane à l'eau distillée stérile à $55^{\circ} \mathrm{C}$

\section{Définition et préparation du milieu RCM modifié}

Le milieu de base est le RCM Agar (Merck) auquel on ajoute de la fuchsine acide $(1 \mathrm{~g} / \mathrm{l})$. Le milieu est stérilisé à $121^{\circ} \mathrm{C}$ pendant $15 \mathrm{~min}$.

(1) Triton X-100 : Merck 11869.

(2) Trypsine $1: 250$ : Difco 0152-15-9.

(3) Support filtre sous pression, $340 \mathrm{ml}$ : Millipore XX4004740.

Membranes : Millipore AAWP04700. 
Au moment de l'emploi, on ajoute dans le milieu en surfusion $\left(45^{\circ} \mathrm{C}\right.$ ) une solution de cyclosérine (dissoute en eau distillée et stérilisée par filtration) de façon à obtenir une concentration finale de $200 \mu \mathrm{g} / \mathrm{ml}$ de milieu.

Le milieu complet est coulé en boîtes de Pétri (diamètre $55 \mathrm{~mm}$ ), et séché avant emploi sous hotte à flux laminaire ou en étuve à $37^{\circ} \mathrm{C}$.

\section{Recouvrement des Clostridum sp sur RCM modifié}

Cet essai a été réalisé sur des suspensions en eau distillée de spores des germes-tests suivants : C. tyrobutyricum (6 souches), $C$. sporogenes, $C$. bifermentans, B. licheniformis, B. cereus.

$1 \mathrm{ml}$ d'une dilution adéquate de la suspension mère est filtré sousvide ; la membrane est ensuite déposée sur RCM modifié et incubée à $37^{\circ} \mathrm{C}$ pendant $48 \mathrm{~h}$. Les résultats des dénombrements sont comparés aux nombres obtenus par inclusion en RCM.

\section{Analyse des laits expérimentalement contaminés}

Du lait cru a été contaminé par une suspension de spores de C. tyrobutyricum (souche C.N.R.Z. 608) à deux concentrations différentes. Après traitement thermique $\left(75^{\circ} \mathrm{C}-15 \mathrm{~min}\right)$ les Clostridium sont dénombrés d'une part par la méthode I.T.G. (méthode MPN en milieu liquide de Bryant Burkey modifié par Bergère), d'autre part par notre méthode (filtration sous pression de $100 \mathrm{ml}$ de lait, dépôt de la membrane sur RCM modifié et incubation pendant $48 \mathrm{~h}$ ).

\section{RESULTATS ET DISCUSSION}

\section{Mise au point du RCM modifié}

Choix du milieu de base

Le milieu de Bryant Burkey modifié par Bergère n'a pas été retenu comme base du milieu recherché ; en effet, sur ce milieu C. tyrobutyricum ne se développe que lentement, son temps de génération étant de l'ordre de 1 h 15 à 1 h 45 (Bergère, 1983). Le milieu RCM (Hirsch et Grinsted, 1954), qui permet une croissance rapide de tous les Clostridium, a été choisi, la nécessaire sélectivité étant recherchée dans l'addition d'antibiotiques.

\section{Elimination des Bacillus sp}

L'addition de cyclosérine, antibiotique connu pour inhiber les anaérobies facultatifs (Hall et coll., 1969, Harmon et coll., 1971), 
utilisé à la dose de $200 \mu \mathrm{g} / \mathrm{ml}$, permet d'éliminer les espèces de Bacillus les plus fréquentes dans le lait, sans entraver la croissance des Clostridium.

Elimination des Clostridium autres que C. tyrobutyricum

Les effets de différents antibiotiques et colorants ont été testés à plusieurs concentrations sur les micro-organismes tests choisis ; $C$. bifermentans et $C$. sporogenes sont aussi, voire plus résistants que $C$. tyrobutyricum à tous les antibiotiques testés. Ces antibiotiques ne permettent donc pas le dénombrement sélectif de $C$. tyrobutyricum, en présence d'autres Clostridium.

\section{Amélioration du contraste colonies-membrane}

Dans l'incapacité de rendre le milieu totalement sélectif, nous avons tenté de le rendre différentiel. A cet effet 6 colorants ont été testés : fuchsine acide, fuchsine basique, rouge neutre, safranine, vert brillant (tous à $1 \mathrm{~g} / \mathrm{l}$ ), cristal violet $(0,01 \mathrm{~g} / \mathrm{l})$.

Un lait cru ensemencé par une suspension de spores de $C$. tyrobutyricum est traité et filtré selon le protocole décrit ci-dessus. Les membranes sont ensuite déposées sur RCM Agar additionné du colorant.

Seule la fuchsine acide donne des résultats intéressants : à la dose utilisée $(1 \mathrm{~g} / \mathrm{l})$, elle n'est pas inhibitrice, mais permet de distinguer les colonies de $C$. tyrobutyricum des colonies des autres espèces de Clostridium testées :

- C. tyrobutyricum : donne des colonies bombées, luisantes, à contours nets, jaune à rose foncé suivant les souches.

- $C$. bifermentans : donne des colonies incolores, plates, à bords diffus, entourées d'un halo "translucide 》 de 2 à $3 \mathrm{~mm}$.

- C. sporogenes donne des colonies rosées, ayant une tendance à l'étalement et moins régulières que celles de $C$. tyrobutyricum.

La fuchsine acide permet donc de dénombrer spécifiquement $C$. tyrobutyricum en présence des autres eșpèces de Clostridium testées.

\section{Recouvrement des Clostridium sp sur RCM modifié}

Les résultats consignés dans le tableau 1 montrent que toutes les espèces de Clostridium testées se développent sur RCM modifié ; les deux espèces de Bacillus testées sont par contre totalement inhibées.

Pour la plupart des suspensions, le dénombrement sur membrane après culture sur RCM modifié donne des résultats inférieurs à ceux obtenus par dénombrement après inclusion en RCM ; ce 
TABLEAU 1

Recouvrement de Clostridium sp sur RCM modifié

\begin{tabular}{l|c|c}
\hline \multicolumn{1}{c|}{ Germe test } & $\begin{array}{c}\text { Témoins } \\
\text { inclusion en RCM } \\
\left({ }^{*}\right)\end{array}$ & $\begin{array}{c}\text { Essais } \\
\text { membrane sur RCM } \\
\text { modifié (*) }\end{array}$ \\
\hline C. tyrobutyricum 500 & 438 & 282 \\
C. tyrobutyricum 510 & 270 & 153 \\
C. tyrobutyricum 596 & 194 & 30 \\
C. tyrobutyricum 602 & 82 & 28 \\
C. tyrobutyricum 603 & 500 & 300 \\
C. tyrobutyricum 608 & 79 & 87 \\
C. sporogenes & 118 & 112 \\
C. bifermentans & 122 & $\begin{array}{c}\text { Absence totale de } \\
\text { développement } \\
\text { Absence totale de } \\
\text { développement }\end{array}$ \\
\hline B. licheniformis & 94 &
\end{tabular}

$\left(^{*}\right) \quad$ Nombre de spores par ml.

phénomène a d'ailleurs été signalé pour de nombreux autres microorganismes dénombrés sur membrane filtrante et pourrait être du au piégeage d'une partie des spores dans la matrice cellulosique de la membrane, les empêchant de former des colonies en surface.

\section{Analyse de laits expérimentalement contaminés}

Les résultats figurent dans le tableau 2.

Dans cette expérience limitée (une seule souche de C. tyrobutyricum testée), le taux de recouvrement des spores par filtration sur membrane et culture sur RCM modifié, est de l'ordre de $100 \%$. Les résultats obtenus sur le même lait par la méthode M.P.N. préconisée par l'I.T.G. sont inférieurs 


\section{TABLEAU 2}

Essais de recouvrement de $C$. tyrobutyricum sur RCM modifié à partir de laits contaminés expérimentalement

\begin{tabular}{l|c|c}
\hline & \multicolumn{2}{|c}{ Nombre de spores/1 de lait } \\
\cline { 2 - 3 } & $\begin{array}{c}\text { Méthode ADRIA } \\
48 \mathrm{~h} 37^{\circ} \mathrm{C}\end{array}$ & $\begin{array}{c}\text { Méthode ITG } \\
7 \text { jours } 37^{\circ} \mathrm{C}\end{array}$ \\
\hline Lait +1640 spores/1 & 1670 & 900 \\
Lait +330 spores/1 & 460 & 240 \\
Lait non ensemencé & 130 & 80 \\
\hline
\end{tabular}

\section{CONCLUSION}

La filtration sous-pression du lait, combinée à l'incubation de la membrane sur RCM gélosé modifié, permet de dénombrer spécifiquement $C$. tyrobutyricum en $48 \mathrm{~h}$, au seuil désiré de 200 spores par litre.

L'application du procédé à des laits contaminés expérimentalement a confirmé l'intérêt de la méthode ; toutefois l'expérimentation n'a porté que sur un nombre de souches et d'espèces trop limité pour permettre d'être tout à fait affirmatif. En outre la méthode nécessite une incubation en jarre d'anaérobiose.

D'autre part une expérimentation en conditions industrielles est maintenant indispensable, mais elle implique sans doute qu'un certain nombre de conditions soient préalablement remplies, notamment la disponibilité commerciale d'un système de filtration simplifié et d'un milieu de culture standardisé.

Enfin ce milieu mi-sélectif, mi-différentiel n'est qu'un pis aller et il serait évidemment souhaitable de disposer d'un milieu parfaitement sélectif de C. tyrobutyricum.

Ces différents points sont actuellement au centre de nos préoccupations.

\section{Remerciements}

Nous sommes reconnaissants au Dr J.L. Bergère pour ses conseils pertinents et pour la fourniture de souches, ainsi qu'au Dr Madeleine Sebald pour plusieurs discussions stimulantes. 
Ces travaux ont été réalisés grâce au soutien financier de la Délégation à la Recherche Scientifique et Technique et de la Fédération Nationale des Producteurs de Lait, dans le cadre d'une convention avec la Communauté Economique Européenne.

\section{Bibliographie}

Bergère (J.L.), HeRmier (J.) (1965). - Etude des facteurs contrôlant la croissance et la sporulation de Clostridium butyricum. Ann. Inst. Pasteur, 109, 80-89.

Bergère (J.L.), Bienassis (G.), Hermier (J.), Mocouot (G.), Gouet (Ph.), Corrot (G.), ZeLTER (\$.Z.) (1972). - Expérimentation sur les possibilités d'utiliser de l'ensilage AIV et de l'ensilage de maïs pour l'affouragement des vaches dont le lait sert à la fabrication de l'Emmental. B.T.I., 266, 29-58.

BERgÈRE (J.L.) (1983). - Méthodes de contrôle de la contamination du lait en butyrique. Journée Nationale d'Information sur « Ensilage et qualité fromagère du lait. Les butyriques ». Fédération Nationale des Producteurs de Lait.

Bourgeors (C.M.), Le Parc (O.), Abgrall (B.), Cleret (J.J.) (1984). - Membrane filtration of milk for counting spores of Clostridium tyrobutyricum. J. Dairy Sci., 67 (11), 2493-2499.

CERF (O.), BeRgèRe (J.L.) (1968). - La numération des spores de Clostridium et son application au lait et aux produits laitiers. II. Numération des différents groupes de Clostridium. Le Lait, 478, 501-519.

GIBSoN (T.) (1965). - Clostridia in silage. J. appl. Bact., 28 (1), 56-62.

Goudkov (A.V.), Sharpe (M.E.) (1965). - Clostridia in dairying. J. appl. Bact., 28 (1), 63-73.

GoUet (Ph.), Bergère (J.L.) (1973). - Ensilage et gonflement butyrique des fromages. Fourrage, 56, 89-137.

HaLl (W.M.), WitZEMAN (J.S.), JANES (R.) (1969). - The detection and enumeration of Clostridum perfringens in foods. J. Food Sci., 34, 212-214.

Harmon (S.M.), KautTer (D.A.), Peeler (J.T.) (1971). - Improved medium for enumeration of Clostridium perfringens. Appl. Microbiol., 22, 688-692.

HIRSCH (A.), GRINSTED (E.) (1954). - Methods for the growth and enumeration of anaerobic spore formers from cheese, with observations on the effect of nisin. J. Dairy Res., 21, 101-110.

WANG (D.I.C.), Scharer (J.), HuMPhrey (A.E.) (1964). - Kinetics of death of bacterial spores at elevated temperatures. Appl. Microbiol., 12 (5), 451-454. 